VIRAL HEPATITIS

\title{
A lack of hepatocyte STING favours HBV infection
}

HBV selectively infects hepatocytes and can cause chronic hepatitis that might lead to hepatocellular carcinoma. Knowledge of how chronic HBV infections are established was previously limited. Research published in Hepatology now provides insight into this process, showing that hepatocytes lack the ability to recognize foreign HBV DNA, thereby hampering the innate immune response.

The recognition of foreign DNA (such as some viruses) is performed by intracellular DNA-sensing proteins, which then activate the stimulator of interferon genes (STING) - ultimately leading to the expression of interferons that contribute to the immune response towards viral infections. However, how HBV has adapted to specifically replicate in hepatocytes and which immune mechanisms might be involved in controlling the virus was not fully understood.
"[My] laboratory has experience in innate immunology to DNA viruses, mainly with a focus on herpesviruses," explains senior author Søren Paludan. As STING had previously shown important roles in controlling herpes and other DNA viruses, and given that an earlier immune response to $\mathrm{HBV}$ infection might lead to a more effective clearance of the virus by the host, the investigators chose to examine the role of the STING pathway in controlling HBV infections.

Using an in vivo mouse model of HBV infection, in which the virus was encoded in an adenoviral vector, the most important finding was that hepatocytes do not express STING and do not induce antiviral interferons in response to foreign DNA. However, when STING was specifically introduced into hepatocytes, DNA-sensing was enabled and led to restricted HBV replication. Furthermore, the researchers found that primary human hepatocytes also $c$

...hepatocytes

do not express

STING and

do not induce

antiviral

interferons...

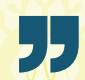

lacked detectable levels of STING and had reduced responsiveness to foreign DNA, validating the results from the mouse model.

"It has been reported that hepatocytes do not respond with strong interferon responses to $\mathrm{HBV}$ infection. We now provide the mechanism underlying the phenomenon," concludes Paludan, which might also explain why HBV has adapted to replicate in hepatocytes. In terms of future work, he believes two options are worthwhile: "One is to understand why hepatocytes have evolved to not respond immunologically to foreign DNA. Another is to explore how other cell types in the liver (resident and infiltrating) sense $\mathrm{HBV}$, and how the virus seeks to evade these responses." lain Dickson

ORIGINAL ARTICLE Thomsen, M. K. et al. Lack of immunological DNA sensing in hepatocytes facilitates hepatitis B virus infection. Hepatology http://dx.doi.org/10.1002/hep.28685 (2016) 